\title{
Superior Mesenteric Arteriovenous Fistula Presenting with Massive Lethal Upper Gastrointestinal Bleeding 14 Years after Small Bowel Resection
}

\author{
Dušan Grujić ${ }^{1}$, Aleksandar Knežević ${ }^{1}$, Svetlana Vojvodić², Bojana Grujić3 \\ ${ }^{1}$ Department of Internal Medicine, Emergency Center, Clinical Center of Vojvodina, Novi Sad, Serbia \\ ${ }^{2}$ Department of Laboratory Diagnostics, Institute for Blood Transfusion of Vojvodina, University of Novi Sad Faculty of Medicine, Novi Sad, Serbia \\ ${ }^{3}$ Department of Anesthesia and Intensive Therapy, Emergency Center, Clinical Center of Vojvodina, Novi Sad, Serbia
}

Background: Arteriovenous fistulas (AVFs) of the superior mesenteric vasculature are rarely encountered. We present a case of an iatrogenic superior mesenteric AVF in a patient who had undergone a small bowel resection 14 years previously.

Case Report: The 45-year-old male was admitted with bloody diarrhea, fatigue, weight loss and moderate ascites. On the fifth hospital day, while being evaluated for suspected liver cirrhosis, the patient developed severe gastrointestinal (GI) bleeding. An upper GI endoscopy revealed bleeding, esophago-gastric varices. A thorough clinical examination pointed to a paraumbilical murmur and thrill, while a contrast enhanced computerized tomography (CT) scan of the abdomen revealed the presence of cystic dilatation of the superior mesenteric vein (SMV), hepatomegaly and ascites.
Duplex ultrasonography and 3D-computed tomography angiography (3D-CTA) confirmed the existence of AVF between the superior mesenteric artery (SMA) and SMV, which was the cause of portal hypertension and variceal bleeding. Despite the fact that AVF was ultimately recognized, uncontrollable hypovolemic shock caused by hematemesis, precluded the probable efficiency of further therapeutic attempts.

Conclusion: Successful management of mesenteric blood vessel AVFs involves timely and the accurate diagnosis and early treatment in order to prevent the development of life threatening complications.

Keywords: Iatrogenic arteriovenous fistula, portal hypertension, superior mesenteric artery, variceal bleeding
Superior mesenteric arteriovenous fistula (SMAVF) is an extremely infrequent vascular disorder. It is characterized by abnormal, direct communication between high-pressure superior mesenteric artery (SMA) and low-pressure superior mesenteric vein (SMV). This shunt allows blood to bypass the intestinal capillary bed and produces hypertension in the portal circulation. Although SMAVF can be congenital, the majority of cases reported have been due to abdominal trauma or iatrogenic causes. These fistulas originate from an undetected injury to SMA and SMV and can present several days to several years later, usually following bowel-related surgery. Manifes- tations of SMAVF include a wide variety of symptoms such as mild abdominal pain, diarrhea and weight loss. Due to the often obscured presence of the fistula, complications related to long-standing arteriovenous (AV) shunt and high portal blood pressure are potentially fatal. The most serious complications are congestive heart failure and portal hypertension, which may lead to gastro-esophageal variceal bleeding (1). General mortality for untreated cases of portal arteriovenous fistula (AVF) is estimated at about $26 \%$ (2). Here, we report a case of delayed presentation of acquired SMAVF in a male adult after small bowel resection.

Address for Correspondence: Dr. Dušan Grujić, Department of Internal Medicine, Emergency Center, Clinical Center of Vojvodina, Novi Sad, Serbia Phone: +381648069636 e-mail: dusan.grujic@kcv.rs

Received: 14.07.2014 Accepted: 04.01.2015 • DOI: 10.5152/balkanmedj.2015.15608

Available at www.balkanmedicaljournal.org 


\section{CASE PRESENTATION}

A 45-year-old man was admitted to the ward complaining of general fatigue, ascites and diarrhea. He was having up to ten stools a day, which were occasionally bloody. The symptoms had persisted for one month, during which he had lost $10-15 \mathrm{~kg}$. In the days prior to arrival at the hospital, the patient noticed slight abdominal swelling. The medical and surgical history was insignificant, other than an abdominal surgery performed 14 years before. The surgery allegedly involved an explorative laparotomy and small bowel resection due to mesenteric ischemia, although no medical documentation was presented.

Clinical examination on the day of admission revealed a pale, undernourished man with a body mass index of 16.3 and without any stigmata of chronic liver disease. The abdomen was mildly distended with an obvious median scar due to the previous laparotomy.

An abdominal ultrasound revealed enlargement of the portal vein (PV), measuring $20 \mathrm{~mm}$ in diameter, increased density of the liver and severe ascites. The initial hemoglobin level was $109 \mathrm{~g} / \mathrm{L}$. Prothrombin time (PT-INR) and activated partial thromboplastin time (APT-INR) international normalized ratios were 1.56 and 1.04 , while the levels of albumin, total bilirubin, alanine transaminase and aspartate aminotransferase were 35.6 $\mathrm{g} / 1,1.05 \mathrm{mg} / 1,34 \mathrm{IU} / 1$ and $33 \mathrm{IU} / 1$, respectively. Serum electrolytes were within normal limits. Evaluation for possible causes of portal hypertension such as alcoholic hepatitis, viral hepatitis, Wilson's disease and portal vein thrombosis was negative.

On the sixth day of hospitalization, the patient developed severe acute gastrointestinal (GI) bleeding. An emergency upper GI endoscopy showed active bleeding from grade III esophageal varices, varices of the cardia and fundus of the stomach as well as evidence of congestive gastropathy. After an unsuccessful attempt of endoscopic therapy, massive bleeding was initially controlled using balloon tamponade with Sengstaken-Blakemore compression tube (SengstakenBlakemore tube, 3 way; Rusch GmbH, Kemen, Germany) and the patient was transferred to the Department of Internal medicine, Emergency center. After the administration of an intravenous bolus dose of Octreotide (Sandostatin; Novartis AG, Basel, Switzerland) continuous infusion at a dose of $50 \mathrm{mcg} /$ hr was initiated. His vital signs were as follows: pulse rate, 120 beats per minute; respiratory rate, 22 breaths per minute; and blood pressure, $130 / 80 \mathrm{~mm} \mathrm{Hg}$. The hemoglobin level fell to $92 \mathrm{~g} / \mathrm{L}$ and the patient's PT-INR and APT-INR were 4.52 and 1.24 , respectively.

More detailed physical examination of the patient's distended abdomen revealed an unusual discrete periumbilical thrill and a

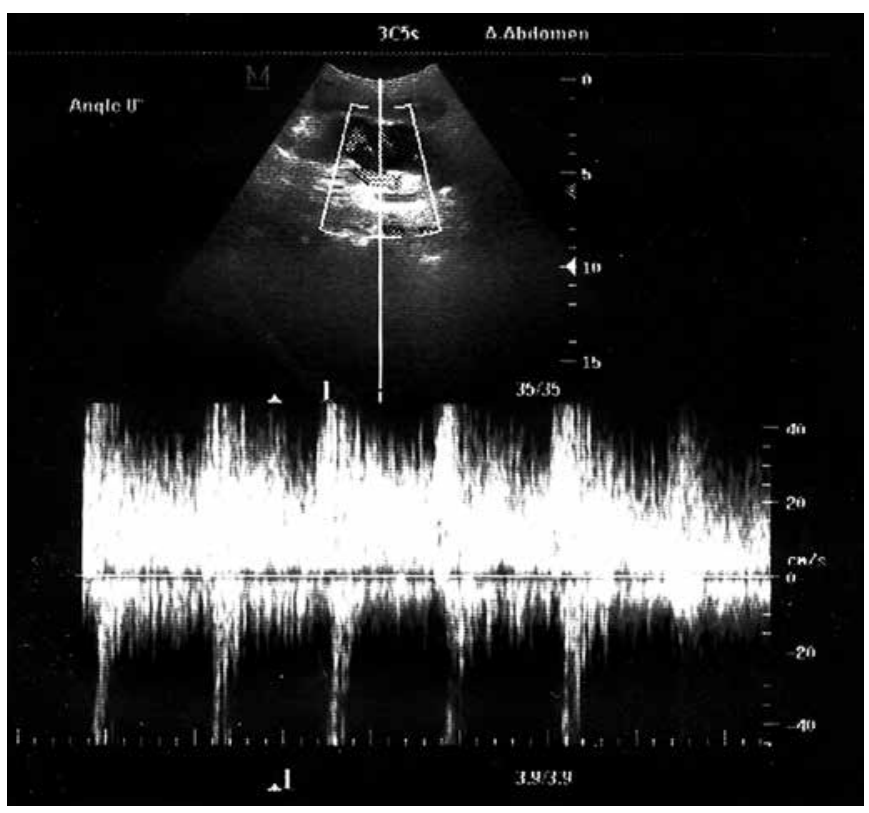

FIG. 1. Duplex ultrasound with high diastolic SMAVF flow

machinery-type bruit over the right mesogastrium. Additionally, duplex ultrasonography was performed which confirmed the suspected existence of SMAVF (Figure 1). Contrast-enhanced computed tomography (CT) scan of the abdomen revealed thickening of small and large bowel walls along with a large aneurismal dilatation of the SMV, measuring $3.2 \mathrm{~cm}$ in diameter (Figure 2). 3D-computed tomography angiography (Somatom Definition Flash; Siemens, Erlangen, Germany) of the abdominal vessels confirmed the existence of AV fistula between the mid-portion of the SMA and SMV (Figure 3). Chest radiograph showed marked cardiomegaly. On the following day, massive variceal bleeding recurred followed by a rapid drop in blood pressure and the development of hypovolemic shock. Due to respiratory arrest, the patient was intubated, resuscitated and transferred to the intensive care unit.

Variceal hemorrhage could not be controlled despite repeated attempts of balloon tamponade and numerous blood transfusions. The patient received a total of 15 units of packed red blood cells. Following several reanimation attempts, the patient died later the same day due to severe hemorrhagic shock.

\section{DISCUSSION}

Since the first report by Movitz and Finne in 1960, only 42 cases of iatrogenic superior mesenteric AV fistula have been documented (present case included), with the majority occurring as a late complication of bowel resection (3-8). The incidence might be underestimated due to unspecific, misleading clinical signs and the often long delay between initial vessel injury and 

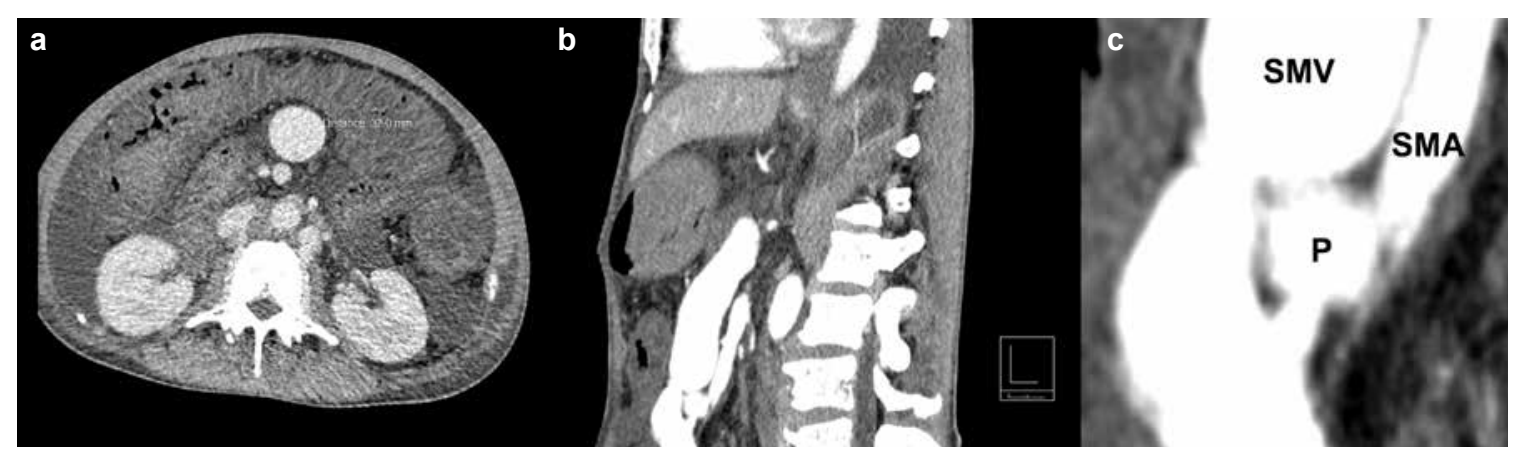

FIG. 2. a-c. Axial contrast-enhanced computer tomography angiography (CTA) of the abdomen showing the dilated superior mesenteric vein (SMV) measuring $32 \mathrm{~mm}$ in diameter (a). Sagittal view demonstrating the aorta (A), superior mesenteric artery (SMA), pseudoaneurysm (P) and its position to the dilated superior mesenteric vein (SMV) $(b, c)$.

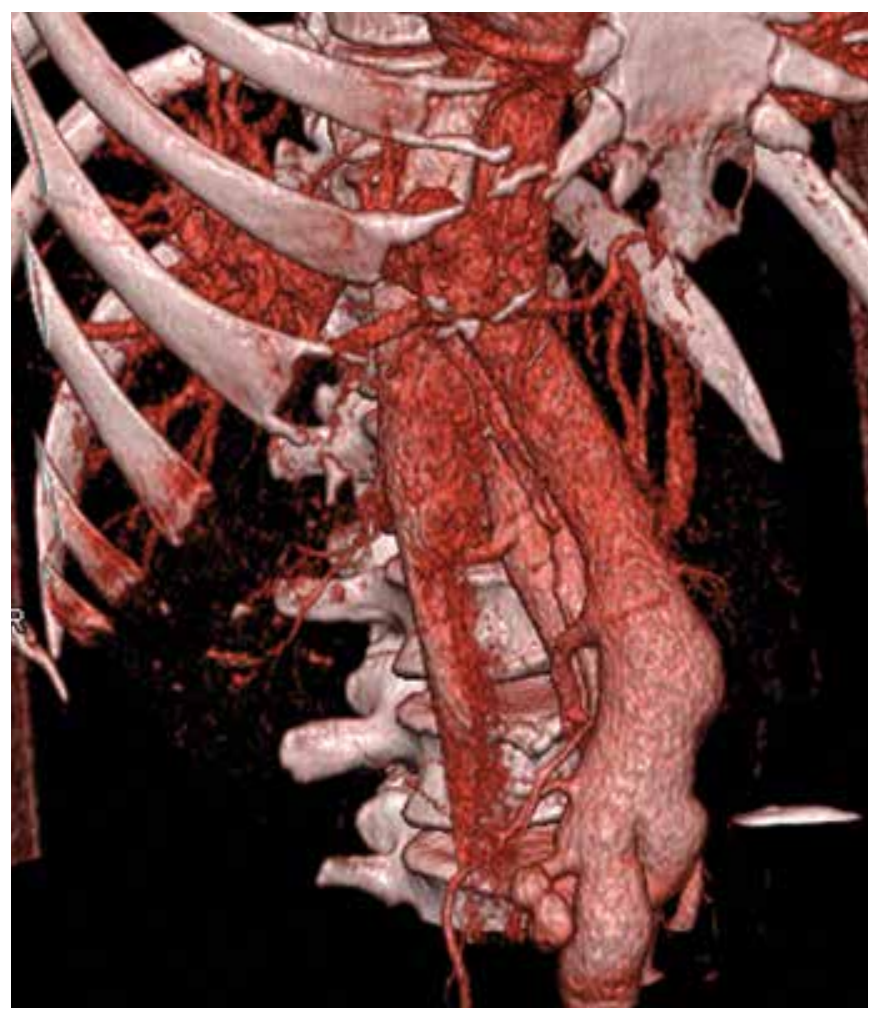

FIG. 3. 3D reconstructed CT angiogram showing aneurismal dilatation of the SMV and PV

diagnosis. Late diagnosis, up to 16 and 20 years after abdominal surgery, has been reported $(9,10)$. In our patient, symptoms of portal hypertension pointed to the diagnosis 14 years after small bowel resection. In several cases, suture material in close relation to the fistula has been found, indicating that a single transfixion suture, which passes through an artery and vein simultaneously, is the probable source of fistula formation (11).

Patients usually present with abdominal pain, diarrhea, recurrent GI bleeding, weight loss, or ascites. Abdominal scar due to previous laparotomy and the discovery of an unexplainable abdominal bruit should raise suspicion of this rare disor- der in a patient with a cirrhotic aspect. When the diagnosis of mesenteric AV fistula is suspected, abdominal duplex ultrasonography can help to identify highly specific high diastolic blood flow in the fistula region and the aneurismal dilatation of the SMV. This was the first test we used in our patient. The most reliable test to confirm the diagnosis and provide the best treatment strategy is contrast enhanced CT angiography. Successful SAMVF treatment relies on direct fistula ligation during an open surgery or endovascular radiological procedures. Previous abdominal surgery and bowel resection with probable adhesions render open surgery more dangerous than endovascular procedures, which should, therefore, be the first treatment choice. Since the first report in 1982, endovascular treatment for iatrogenic SMAVF has been reported in 16 cases (6). Coil embolization was successfully used 15 times, and vascular plug insertion was performed twice $(5,6,12,13)$. Embolization of the fistula is not a risk-free procedure, since occluding other branches of the artery can produce mesenteric infarction. Coil migration, leading to portal vein thrombosis, is an important reported complication of the procedure (14). The goal of the treatment is to cure pre-hepatic portal hypertension and prevent associated complications. Treatment is often performed in an emergency setting, since a significant number of these patients will present with advanced portal hypertension and upper GI bleeding due to esophageal varices. Patient's poor general health and the torrential nature of bleeding are mainly responsible for the dismal prognosis of variceal bleeding. Failure to control bleeding or early rebleed within 5 days still occurs in $15 \%$ to $20 \%$ of patients with acute variceal bleeding, based on data from cirrhotic patients. Mortality in this group of patients is up to $50 \%$ (15). Late referral to the hospital, resulting in long-standing portal hypertension and AV shunt, affected our patient's intestinal and cardiac functions. Malnutrition and multiple organ dysfunction predisposed this patient to increased mortality following the first variceal bleeding episode. Due to ongoing bleeding, 
despite volume replacement, transfusions and vasoactive drug therapy, sufficient hemodynamic stabilization could not be achieved. This made different treatment modalities for closure of the fistula unfeasible.

In conclusion, SAMF is a rare complication following injury to mesenteric blood vessels. Signs of portal hypertension accompanied by normal or mildly affected liver function tests and a history of abdominal surgery must alert physicians to this rare complication. Only early diagnosis and the opportune treatment of mesenteric blood vessel AVF can prevent a potentially fatal outcome.

\section{Ethics Committee Approval: N/A.}

Informed Consent: Written informed consent was obtained from the patient.

Peer-review: Externally peer-reviewed.

Author contributions: Concept - D.G.; Design - D.G., A.K., S.V.; Supervision - A.K., S.V., B.G.; Resource - D.G., B.G.; Materials D.G., A.K., B.G.; Data Collection \&/or Processing - D.G., B.G.; Analysis \&/or Interpretation - D.G., A.K., S.V., B.G.; Literature Search - D.G., B.G.; Writing - D.G., S.V., B.G.; Critical Reviews D.G., A.K., S.V.

Acknowledgements: The authors would like to thank to all personnel of the department for their help.

Conflict of Interest: No conflict of interest was declared by the authors.

Financial Disclosure: The authors declared that this study has received no financial support.

\section{REFERENCES}

1. Kato S, Nakagawa T, Kobayashi H, Arai E. Superior mesenteric arteriovenous fistula: report of a case and review of the literature. Surg Today 1993;23:73-7. [CrossRef]

2. Van Way CW 3rd, Crane JM, Riddell DH, Foster JH. Arteriovenous fistula in the portal circulation. Surgery 1971;70:876-90.

3. Xu CE, Guo L, Li GX, Zhang SG, Yu ZH, Ruan CL. A rare case of superior mesenteric arterio-venous fistula causing jejunal varices and recurrent GI bleeding. EJVES Extra 2006;12:35-7. [CrossRef]

4. Hussein M, Issa G, Muhsen S, Haydar A. Superior mesenteric arteriovenous fistula embolisation complicated by bowel ischaemia. BMJ Case Rep 2013;2013.

5. Temin N N, Flacke S, Ahari H K. Superior Mesenteric Arteriovenous Fistula Imaging Findings and Endovascular Treatment. Vasc Endovascular Surg 2012;46:675-8. [CrossRef]

6. Shintani T, Mitsuoka H, Masuda M. Transcatheter coil embolization of an iatrogenic superior mesenteric arteriovenous fistula: report of a case. Surg Today 2011;41:556-9. [CrossRef]

7. Bratton C F, Hamid A, Selby J B, Baliga P K. Case report: gastrointestinal hemorrhage caused by a pancreas transplant arteriovenous fistula with large psuedoanuerysm 9 years after transplantation. Transplant Proc 2011;43:4039-43. [CrossRef]

8. Buttarelli L, Capocasale E, Marcato C, Mazzoni M P, Iaria M, Rossi C. Embolization of pancreatic allograft arteriovenous fistula with the Amplatzer vascular plug 4: case report and literature analysis. Transplant Proc 2011;43:4044-47. [CrossRef]

9. Diehl J T, Beven E G. Arteriovenous fistulas of the mesenteric vessels. Report of a case and review of the literature. J Cardiovasc Surg (Torino) 1982;23:334-7.

10. Popović DDj, Spuran M, Davidović L, Alempijević T, Ugljesić M, Banzić I, et al. Portal hypertension caused by postoperative superior mesenteric arteriovenous fistula. Vojnosanit Pregl 2012;69:623-6. [CrossRef]

11. Durham M W, Robnett A H, Harper H P, Yekel R. Arteriovenous fistula of the mesenteric vessels. West J Surg Obstet Gynecol 1962;70:9-11.

12. Wong KK, Wong JSW, Tso WK, Tai KS. Superior Mesenteric Arteriovenous Fistula: an Unusual Cause of Ascites. J HK Coll Radiol 2007;10:77-80.

13. Eiberg JP, Bundgaard-Nielsen M, Hansen MA, Secher NH. Endovascular repair of iatrogenic superior mesenteric arteriovenous fistula. EJVES Extra 2009;17:21-3. [CrossRef]

14. Mick SL, Bush HL Jr, Barie PS. Superior mesenteric arteriovenous fistula causing massive hematemesis. Surgery 2003;134:102-4. [CrossRef]

15. Ba-ares R, Albillos A, Rincón D, Alonso S, González M, Ruizdel-Arbol L, et al. Endoscopic treatment versus endoscopic plus pharmacologic treatment for acute variceal bleeding: A meta-analysis. Hepatology 2002;35:609-15. [CrossRef] 